Canadian Oncology

Nursing Journal

Revue canadienne

de soins infirmiers

en oncologie

Volume 28, Issue 1 • Winter 2018

elSSN: 2368-8076 


\title{
Assessing the application of the Synergy Model in hematology to improve care delivery and the work environment
}

\author{
by Georgia Georgiou, Yayra Amenudzie, Enoch Ho, and Elizabeth O’Sullivan
}

\begin{abstract}
A pilot project was undertaken to evaluate the impact of the Synergy Model (Curley, 1998) on patient care delivery and professional practice in a hematology unit. Patient characteristics were matched to nurse competency when making the nursing assignments and acuity scores were used to make staffing adjustments. The model resulted in "better fit" assignments, with 87\% of nurses reporting their competencies were well matched with patient acuity, compared to $48 \%$ before the model implementation. Nurse satisfaction regarding the level of support for novice nurses, involvement in nursing assignments, workload, and engagement also improved. Reduction in safety occurrences and overtime were also observed. The Synergy Model offers a promising framework for improving care delivery and the practice environment in other similar patient populations.
\end{abstract}

\section{BACKGROUND}

Tn 2012, The Hematology/Hematopoietic Stem Cell 1 Transplant (HSCT) Program at the Juravinski Hospital and Cancer Centre identified a unique opportunity to enhance patient care and the work environment by piloting the Synergy Model (Curley, 2007). This model is a professional practice framework that involves assessing patient acuity and making assignments based on a "fit" between patient needs and staff competency. The unit was struggling with teamwork, communication, and with making the assignments for registered nurses and health care aides (HCAs). The process for making the daily assignment was largely based on habit, geography, intuition, and consideration of the time required to accomplish diverse interventions. As a result, practices were

\section{ABOUT THE AUTHORS}

Georgia Georgiou, BA, Med, Clinical Manager Stem Cell, Ron and Nancy Clark Hematology Unit, Juravinksi Hospital and Cancer Centre, Hamilton Health

Sciences, 699 Concession Street, Hamilton, ON L8V 5C2.

T: 905-521-2100 ext. 43910; Email: georgg@hhsc.ca

\section{Transplant Program:}

Yayra Amenudzie, RN, MN, Hematology Staff Nurse, Ron and Nancy Clark Hematology Unit, Juravinksi Hospital and Cancer Centre, Hamilton Health Sciences, Hamilton, ON.

Email: amenudzie@hhsc.ca

Enoch Ho, MPh, RPT, RAcu, Quality \& Value Improvement Office, Ron and Nancy Clark Hematology Unit, Juravinksi Hospital and Cancer Centre, Hamilton Health Sciences, Hamilton, ON.

Email: hoenoch@HHSC.CA; T: 905-521-2100 ext. 42097

Elizabeth O'Sullivan, RN, BScN, MScN, Retired Director inconsistent across shifts and charge nurses. This was causing friction amongst the team and complaints about workload and unsafe assignments. These challenges were negatively affecting teamwork, staff morale, the quality of patient care, patient and staff satisfaction, recruitment, and retention.

In searching for solutions, the Synergy Model stood out as a promising framework. The model is purported to offer an effective way for organizing the work of patient care, such that patient outcomes are optimized, and a "magnet" work environment is created that helps attract and retain nurses (Kaplow \& Reed, 2008). MacPhee, Wardrop, Campbell, and Wejr (2011) found that the model “ ... helped clarify who was best suited to provide care for patients with particular needs; the model's patient-centred philosophy enhanced collaboration and teamwork around the common goal of better care provision, and the model and tool provided staff with a unified language, a systematic scoring approach and objective staffing guidelines"(p. 52). In addition, the model has been shown to be effective in improving care delivery and patient outcomes in a children's hospital (Gralton, 2012), as well as patient satisfaction and performance of nurses in cardiac intensive care units (Khalifehzadeh, 2012).

The Synergy Model has been applied in different healthcare settings (acute, residential, and community) and with different patient populations. The Model is designed to help healthcare providers prioritize patient care, identify workloads associated with caseloads, provide a systematic approach to rounds and case conferences, train and orientate new staff, make treatment planning decisions, and make real-time care delivery and staffing assignments based on synergy scores (MacPhee, Wardrop, Campbell, \& Wejr, 2011; Kerfoot et al., 2006; Swickard et al., 2015; Carter \& Burnette, 2011). However, we could not find any applications of the model in an acute hematology inpatient unit.

\section{PURPOSE}

The purpose of the pilot project was to examine the adaptability, applicability, and effectiveness of the Synergy Model in a Canadian inpatient hematology/HSCT setting. The pilot was conducted in two phases over the course of 18 months.

Phase 1 was designed to answer the following questions:

- Can the synergy model be adapted for a hematology patient population?

- Is it possible to implement the model in an acute inpatient setting? and

- What are the critical success factors and limitations of the model? 
The results from Phase 1 demonstrated that the model can be adapted and successfully implemented in a hematology patient population. The Provincial Nursing Workload Project Resource Toolkit published by the British Columbia Nurses' Union (2010) was used to develop a patient acuity tool for our patient population, which included four patient characteristics: complexity, stability, participation in care, and predictability. Furthermore, the processes that were developed for scoring patients and using the scores to make the nursing assignment and decisions about staffing adjustments, proved feasible to implement in a Canadian acute inpatient setting. The findings from phase one, including lessons learned about key enablers and limitations of the model, have been described elsewhere (Amenudzie, Georgiou, Ho, \& O’Sullivan, 2017).

This paper focuses on the second phase of the pilot, which was designed to evaluate the usefulness and impact of the synergy model on the professional practice environment and care delivery. The methodology that was used to evaluate the model's usefulness, the findings, recommendations for administrators, and future directions are discussed.

\section{METHODS}

To evaluate the model's impact on the work environment and nursing practice, all nurses were invited to complete a Synergy Work Environment Survey, pre- and post-pilot implementation. The survey included in the Provincial Nursing Workload Project Resource Toolkit (BCNU, 2010) was adapted and used to measure several aspects of the practice environment including:

a) support provided to novice nurses,

b) availability of experienced charge nurses,

c) ability to focus on nursing tasks,

d) ability to practise evidence-based nursing care,

e) collaboration between nurses and other health professionals in decision-making,

f) ability to plan and attend patient care rounds,

g) involvement in resource allocation and utilization decisions,

h) participation in determining staff assignments,

i) extent to which patient assignments match nurse competency and enable nurses to provide safe, competent and ethical care,

j) orientation provided to new positions,

k) opportunities for in services, continuing education, debriefing and reflection on practice, and

1) the extent to which the work environment enables nurses to develop a therapeutic and rewarding relationship with patients.

The Chi Square test was used to compare pre- and post-ratings for statistical significance $(\mathrm{p}<0.05)$.

To evaluate the model's impact on nurse satisfaction with the assignment and the degree of fit between RN competencies and patient characteristics, a Nurse Assessment of Patient Assignment survey was developed and completed daily by nurses, over 73 day shifts, from January 13, 2014-March 31, 2014. At the end of every day shift, nurses were asked the following questions about their assignment:
- their competency level (novice, advanced beginner, competent, proficient, expert nurse),

- pace and workload (ideal work pace enabling to go beyond routine care; busy but manageable; busy barely manageable; unmanageable and unsafe),

- whether the synergy scores accurately reflect the patient's needs and acuity,

- the degree of fit between their competency and patient acuity, and

- nurses could also comment on any other issues related to their assignments.

To determine the model's impact on staff engagement, four questions were selected from a hospital-wide staff engagement survey that had been implemented in 2011. The questions measured staff perceptions of feeling engaged, the quality of patient care, work-life balance, and staff Stress Satisfaction Offset Scores. These questions were included in a post-Synergy Assessment survey that was administered at the end of the pilot. Responses from the original hospital-wide engagement survey were filtered to obtain nurse ratings only, and the pre- and post-engagement ratings were compared using the Chi Square Test.

To explore the impact on nurse-sensitive indicators such as patient falls, medication errors, and other safety occurrences, data were generated from the hospital's Safety Occurrence Report (SOR) database. The number of SORs preceding the pilot (Nov 2012-March 2013) was compared to the number of occurrences during the pilot (November 2013-March 2014).

Finally, to assess the impact on resource utilization, the number of shifts when an additional nurse and/or health care aide was needed was tracked. Monthly overtime rates from April 2012 to March 2013 were obtained from the hospital database.

\section{RESULTS}

The pilot was implemented from October 2013-March 2014. A comparison of pre-post measures revealed statistically significant improvements in several areas.

Regarding the usability and usefulness of the Synergy Tool (see Tables 1 and 2), results indicated a better "fit" between patient needs and nurse competency, novice nurses felt better supported in practice, and nurses perceived the patient assignment as more manageable in terms of workload. There was high compliance with applying the tool to score patient acuity and high compliance with use of the tool to create daily nursing assignment and staffing guidelines. In essence, the Synergy scores reflected patients' condition accurately.

\begin{tabular}{|c|c|c|c|}
\hline $\begin{array}{l}\text { RN Ratings of } \\
\text { Assignment }\end{array}$ & \begin{tabular}{|l} 
PRE \\
SYNERGY \\
N = 127
\end{tabular} & $\begin{array}{l}\text { POST } \\
\text { SYNERGY } \\
N=568 \\
\end{array}$ & $\begin{array}{l}\text { Chi } \\
\text { Square } \\
\text { test }\end{array}$ \\
\hline $\begin{array}{l}\text { The majority of RNs on } \\
\text { a shift }(80 \%) \text { rated their } \\
\text { assignment as "Ideal" or } \\
\text { "busy but manageable" }\end{array}$ & $53 \%$ & $70 \%$ & $P<0.05$ \\
\hline
\end{tabular}




\begin{tabular}{|c|c|c|c|}
\hline RN SURVEY - \% of Positive Scores & $\begin{array}{l}\text { PRE SYNERGY N=29; } \\
53 \% \text { Response Rate }\end{array}$ & $\begin{array}{l}\text { POST SYNERGY N=32; } \\
60 \% \text { Response Rate }\end{array}$ & $\begin{array}{l}\text { Chi Square } \\
\text { Test }\end{array}$ \\
\hline Nurses are involved in determining staff assignments & $17 \%$ & $65 \%$ & $P<0.05$ \\
\hline Nurses' skills and expertise are matched with patient care needs & $48 \%$ & $87 \%$ & $P<0.05$ \\
\hline $\begin{array}{l}\text { Novice nurses are supported in practice by accessible, expert and } \\
\text { experienced nurses }\end{array}$ & $55 \%$ & $87 \%$ & $P<0.05$ \\
\hline $\begin{array}{l}\text { Patient assignments are based on nurses' ability to provide safe, } \\
\text { competent, ethical care }\end{array}$ & $34 \%$ & $65 \%$ & $P<0.05$ \\
\hline \multicolumn{4}{|l|}{ Usability of the tool } \\
\hline \multicolumn{3}{|l|}{ Percentage of time patients are rated using the tool } & $100 \%$ \\
\hline \multicolumn{3}{|l|}{ Percentage of time the tool is used to make the nursing assignment } & $100 \%$ \\
\hline \multicolumn{3}{|l|}{ Percentage of time the staffing guidelines are followed } & $76 \%$ \\
\hline \multicolumn{3}{|l|}{ Percentage of time Synergy scores reflected patient acuity and condition } & $94 \%$ \\
\hline
\end{tabular}

\begin{tabular}{|l|}
\hline Table 3: Nurse Ratings of Engagement \\
\hline Staff Engagement Survey Results (Percentage of Positive Scores) \\
\hline Perception of feeling engaged \\
\hline Perception of the quality of patient care \\
\hline Perception of workload and work life balance \\
\hline Stress Satisfaction Offset Scores \\
\begin{tabular}{|l|l|}
\hline Table 4: Impact on Safety Occurrences/Errors \\
\hline $\begin{array}{l}\text { Pre-Synergy Nov } 2012 \text { - } \\
\text { March 2013 }\end{array}$ \\
\hline 144 Safety Occurrence Reports & $\begin{array}{l}\text { 107 Safety Occurrence Reports } \\
\text { March 2014 }\end{array}$ \\
\hline * Reduction in falls, and laboratory incidents \\
\hline
\end{tabular}
\end{tabular}

In terms of the impact on staff engagement (see Table 3), we observed greater nurse engagement and involvement in decision-making and a perceived improvement in the quality of patient care. Nurses reported feeling a better work-life balance.

The impact on safety occurrences/errors (see Table 4) resulted in a reduction in safety occurrences, most notably in patient falls and laboratory errors.

Finally, the impact on resource utilization (see Tables 5 and 6) was that Health Care Aide Resources and Nursing Resources were needed for 27 and 23 shifts respectively. Overtime reduced by $40 \%$ with less monthly variation.

\section{DISCUSSION}

Our team's journey with the Synergy Model and the findings from this pilot project, affirm that the model shows promise in improving care delivery and the work environment. The model helped our team to clarify who is best suited to provide care for patients. It enhanced collaboration and teamwork, provided our team with common language for discussing patient acuity and care needs, and offered a systematic approach for making assignments and staffing decisions. Our experience with the Synergy Model has not only shaped how we make care delivery, resource utilization, and staffing decisions, but it has also

\begin{tabular}{|c|c|c|}
\hline $\begin{array}{c}\text { PRE SYNERGY } \\
\mathbf{4 6 \%} \text { Response Rate }\end{array}$ & $\begin{array}{c}\text { POST SYNERGY } \\
\mathbf{6 0 \%} \text { Response Rate }\end{array}$ & $\begin{array}{c}\text { Chi Square } \\
\text { test }\end{array}$ \\
\hline $43 \%$ & $62 \%$ & $\mathrm{P}<0.05$ \\
\hline $62 \%$ & $76 \%$ & $\mathrm{P}<0.05$ \\
\hline $24 \%$ & $50 \%$ & $\mathrm{P}<0.05$ \\
\hline $26 \%$ & $56 \%$ & $\mathrm{P}<0.05$ \\
\hline
\end{tabular}

\begin{tabular}{|l|c|}
\hline Table 5: Resource Utilization \\
\hline Type of Resource & $\begin{array}{c}\text { Number of Shifts \& Hours } \\
\text { (January 2014-March 2014) }\end{array}$ \\
\hline Health Care Aide & 27 shifts; 303.75 hours \\
\hline Registered Nurse & 23 shifts; 258.75 hours \\
\hline
\end{tabular}

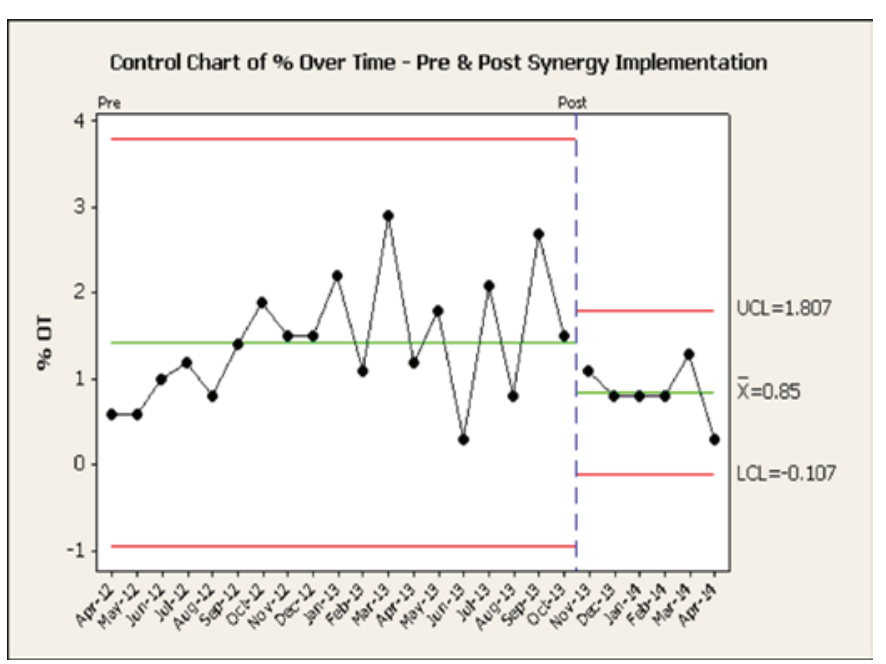

Table 6: Percentage of Overtime

reconfigured how we assess learning needs of nurses, and how we orient and train new staff. The nurse competency assessment is integrated into the staff performance review and professional development process, and the profile of nurse competency is used to determine in which rotations to place new nurses to achieve an optimal skill mix on every shift. 


\section{Implications for Administrators}

The 2006 World Health Report, as well as research on magnet hospitals, highlight the importance of creating a healthy work environment (Havens \& Aiken, 1999; Shamian \& El-Jardali, 2007). Healthy work environment characteristics are linked to nurse satisfaction and the quality of care (Armstrong \& Laschinger, 2006; Laschinger et al., 2003). One important component of a healthy work environment is that front-line nursing staff are engaged in decision-making about the care delivery model. The application of the Synergy Model on our unit increased clinical autonomy and nurse engagement by involving nurses in decisions that impact patient care, the quality of the work environment, and resource utilization. Our results demonstrate that leaders can positively influence practice environments by implementing a framework that embraces a philosophy, structures, and processes that support nurses' control over their practice environment and promotes professional decision-making at the patient and unit level.

Additionally, the Synergy Model aligns well with several Accreditation Canada Cancer and Oncology Service standards, and can be used as evidence that the organization a) provides support to the team to deliver quality cancer care to clients, b) the organization has defined criteria used to assign team members to clients in a fair and equitable manner, and c) team members have input on work and job design.

Finally, the Synergy Model can be readily applied to assess nurse competency and support professional development, which contribute to the creation of a magnet work environment.

\section{Directions for Future Research}

Although our pilot demonstrated a reduction in overtime, safety occurrences, and improvements in nurse engagement and satisfaction, the inter-dependency and complexity of clinical

\section{REFERENCES}

Amenudzie, Y., Georgiou, G., Ho, E., \& O’Sullivan, E. (2017). Adapting and applying the Synergy Model on an inpatient heamatology unit. Canadian Oncology Nursing Journal, 27(4), 338-342.

Armstrong, K., \& Laschinger (2006). Structural empowerment, magnet hospital characteristics, and patient safety culture: Making the link. Journal of Nursing Care Quality, 21(2), 124-132.

British Columbia Nurses' Union. (2010). Provincial nursing workload project resource toolkit for teams: Nursing workload and staffing plan processes. https://www.bcnu.org/Safeworkplace/ DefendProfessionalPractice/Documents/pnwp_resource_toolkit.pdf

Carter, K.F., \& Burnette, H.D. (2011). Creating patient-nurse synergy on a medical-surgical unit. Medsurg Nursing, 20(5), 249-254.

Curley, M.A. (Ed.). (2007). Synergy: The unique relationship between nurses and patients. Indianapolis: Sigma Theta Tau International.

Curley, M.A. (1998). Patient-nurse synergy: Optimizing patient's outcomes. American Journal of Critical Care, 7(1), 64-72.

Gralton, K.S., \& Brett, S.A. (2012). Integrating the synergy model for patient care at Children's Hospital in Wisconsin. Journal of Pediatric Nursing, 27, 74-81.

Havens, D., \& Aiken, L. (1999). Shaping systems to promote outcomes: The magnet hospital model. Journal of Nursing Administration, 29, 14-20.

Kaplow, R., \& Reed, K. (2008). The AACN synergy model for patient care: A nursing model as a force of magnetism. Nursing Economics, 26(1), 17-25. operations makes it impossible to attribute the positive changes to the Synergy Model alone. The Model was implemented concurrently with other interventions designed to improve the work environment. Further investigation is needed to evaluate the impact and cost-effectiveness of this model in relation to staff, patient, and organizational outcomes such as absenteeism, staff turnover, safety occurrences, patient satisfaction, and nurse-sensitive clinical indicators. Finally, there may be value in developing one aggregate score that captures the acuity level overall on the unit. This would allow clinical areas to look at trends in acuity and could potentially simplify the process for determining when staffing adjustments are needed.

\section{CONCLUSIONS}

The Synergy Model has become the operating system for organizing nursing and health care aid work on our unit. It offers an effective framework for improving professional practice and promoting a healthy work environment. The model has enhanced nursing practice by providing a clearer path for professional development and for matching unique patient needs with nurse competency. The flexible nature of the model is well suited to the fluctuating acuity of a hematology patient population and it enables the team and healthcare administrators to make more informed, real-time decisions about resource utilization and allocation in response to changes in patient acuity.

\section{ACKNOWLEDGEMENTS}

We would like to thank our project team, the staff and physicians of the hematology/HSCT unit and the Juravinski Cancer Centre Foundation and F. Hoffmann-La Roche Ltd., which provided funding and support for this pilot project.

Kerfoot, K.M., Lavandero, R., Cox, M., Triola, N., Pacinin, C. \& Hanson, D.M. (2006). Conceptual models and the nursing organization. Implementing the AACN synergy model for patient care. Nurse Leader, 20-26.

Khalifehzadeh, A., Jahromi, M.K., \& Yazdannik, A. (2012). The impact of the Synergy Model on nurses' performance and the satisfaction of patients with acute coronary syndrome. Iranian Journal Nursing and Midwifery Research, 17(1), 16-20.

Laschinger, H., Almost, J., \& Tuer-Hodes. (2003). Workplace empowerment and magnet hospital characteristics. Journal of Nursing Administration, 33(7/8), 410-422.

MacPhee, M.K., Jewell, K., Wardrop, A., Ahmed, A., \& Mildon, B. (2010). British Columbia's provincial nursing workload project: Evidence to empowerment. Canadian Journal of Nursing Leadership, 23(1), 54-63.

MacPhee, M., Wardrop, A., Campbell, C., \& Wejr, P. (2011). The synergy professional practice model and its patient characteristics tool: A staff empowerment strategy. Nursing Leadership, 24(3), 42-55.

Shamian, J., \& El-Jardali, F. (2007). Healthy workplaces for health workers in Canada: Knowledge transfer and uptake in policy and practice. Healthcare Papers, 7(Special Issue), 6-25.

Swickard, S., Swickard, W., Reimer, A., Lindell, D., \& Winkelman, C. (2015). Adaptation of the AACN synergy model for patient care to critical care transport. Critical Care Nursing, 34(1), 16-28. 